\title{
Statistics of Transverse Mode Turn-On Dynamics in VCSEL's
}

\author{
J. Dellunde, M. C. Torrent, J. M. Sancho, and K. A. Shore
}

\begin{abstract}
The turn-on process of a multimode VCSEL is investigated from a statistical point of view. Special attention is paid to quantities such as time jitter and bit error rate. The single-mode performance of VCSEL's during current modulation is compared to that of edge-emitting lasers.
\end{abstract}

Index Terms - Laser modulation, switching dynamics, VCSEL'S.

\section{INTRODUCTION}

$\mathbf{I}$ N RECENT years, quite dramatic improvements have been made in the performance capabilities of vertical-cavity surface-emitting lasers (VCSEL's) and consequently serious consideration can now be given to deploying such devices in a range of practical applications [1], [2]. In this case, it becomes necessary to pay attention to relatively detailed aspects of the device behavior which may impact upon the device operating characteristics. Of concern in many situations is the detailed form of the transverse mode pattern in the device. Considerable attention has been given to elucidating the conditions for the selection of low-order transverse modes and laser designs have been advanced for the promotion of single-transversemode operation [3]-[6]. In the static regime, the prediction of the transverse mode properties is a quite complicated problem requiring account to be taken of details of the device structure and operating conditions and thus can require a rather comprehensive modeling procedure [7]. On the other hand, it is known that spatial hole burning (SHB) and carrier diffusion play key roles in transverse mode selection and, in turn, these processes depend upon the laser drive current [8], [9]. Experiments have been carried out recently showing such effects [10]. Consequently, the excitation of transverse modes when the laser is subject to large excursions in the bias current will be strongly influenced by SHB effects. The laser turn-on regime is a particularly important situation where transverse mode excitation can be expected to be dependent upon relatively large changes in the laser drive current and, moreover, the mode excitation process in that regime needs to be treated dynamically. The aim of the present contribution

Manuscript received September 30, 1996; revised March 10, 1997. This work was supported by Comisión Interministerial de Ciencia y Tecnología under Project PB93-0769-C02-01 and the program Human Capital \& Mobility CIIRX-CT93-0331.

J. Dellunde and J. M. Sancho are with the Departament d'Estructura i Constituents de la Matèria, Facultat de Física, Universitat de Barcelona, E08028 Barcelona, Spain.

M. C. Torrent is with the Departament de Física i Enginyeria Nuclear, EUETIT, Universitat Politècnica de Catalunya, E-08222 Terrassa, Spain.

K. A. Shore is with the School of Electronic Engineering and Computer Systems, University of Wales, Bangor LL57 1UT, Wales, U.K.

Publisher Item Identifier S 0018-9197(97)04708-8. is to offer a tractable method for describing the transverse mode selection process during the laser turn-on regime. The approach adopted here can be seen to be analogous to that used in earlier treatments of longitudinal side-mode excitation in edge emitting semiconductor lasers [11]-[14]. The requirement is for a methodology which permits an evaluation of a sidemode suppression ratio (SMSR) (here for transverse modes) and which, crucially, is also capable of providing an accurate estimate of the relevant statistics of the turn-on process. This is rather a stringent requirement since it is necessary not only to undertake simulations of the dynamical evolution of the identified transverse modes but it is also required that simulations are performed of a large number of turnon processes (typically 10000 turn-on events) in order to extract the required statistical information. It is clear that in order to make reasonable demands upon computer resources an analytical approach must be adopted for this purpose. It is recognized that the use of such an analytical approach inevitably implies that some detailed features of the device behavior may be lost in the modeling process. The technique developed here is, however, sufficiently flexible that further detail of the device characteristics can be incorporated if required. Essentially, such additional features are included by adding further terms to a functional expansion over a basis set. The compromise to be made is then between the relative importance of the particular device feature and the additional effort which is needed to assess its significance in the turnon process. In the results obtained here attention is centered on the influence of SHB and carrier diffusion in determining turn-on events.

The objective of this paper, therefore, is to describe and apply a technique for obtaining an analytical description of transverse mode competition effects in a representative VCSEL structure and, in this way, to facilitate a statistical treatment of mode competition during the turn-on process. The validity of the analytical approach is established by comparison with full numerical treatments of the turn-on events. The structure of this paper is as follows. In Section II, the main features of the dynamical model of the chosen VCSEL structure are described. In Section III, analytical calculations are applied to the calculation of a bit error rate associated with transverse mode excitation. Results obtained from the model are given in Section IV. Conclusions from the work are collected in Section V.

\section{MODEL}

The model utilized in the work reported here incorporates both spatial dependence of carrier and optical field profiles. 


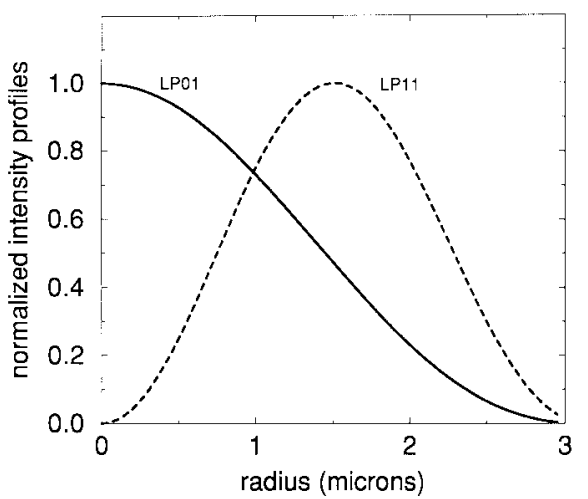

Fig. 1. Normalized radial intensity profiles of the $\mathrm{LP}_{01}$ (solid line) and degenerate $\mathrm{LP}_{11}$ (dashed line) modes of the VCSEL structure.

In this respect, the model is seen as an extension of earlier work on VCSEL transverse mode dynamics [8], [9], [15], [16]. The principal components of the dynamical model are a carrier continuity equation and appropriate rate equations for the mode amplitudes. Underlying these equations are selfconsistent calculations of steady-state modal properties of the chosen structure [5], [6]. For definiteness, attention is focused on a generic VCSEL structure with circular symmetry in which, in general, account is taken of variations in carrier density as a function of radial distance, azimuthal angle, and time. Such a model is appropriate to VCSEL designs of the kind studied, for example, by Ogura [17] and Iga [18]. Device structural parameters have been chosen in accordance with such device geometries. For the model developed here, the case of competition between two modes is treated explicitly but the method can, if required, be extended to account for competition between, for example, an arbitrary number of transverse modes.

It is appropriate to utilize a cylindrical coordinate system $(r, \phi, z)$ to describe the spatial distribution of carriers and optical fields in the structure. The current density distribution $j_{0}(r, \phi, t)$ has been chosen to be azimuthally uniform over a circular disc so that $j_{0}(r, \phi, t)=j_{0}(t)$ for $r<s$ and $j_{0}(r, \phi, t)=0$ otherwise. The active region of the device is taken to be of thickness $d$ and radius $a$. The laser cavity is defined by two highly reflecting mirrors separated by a distance $L$ along the longitudinal axis. The dielectric constants of the core and cladding layers are denoted by $\varepsilon_{1}$ and $\varepsilon_{2}$. In the case of transverse mode competition in a weakly index-guided structure, the appropriate modes for the assumed VCSEL structure are conventionally denoted as the $\mathrm{LP}_{m n}$ modes [19]. The transverse variation of the field of the $(m, n)$ mode is given by

$$
\begin{aligned}
& \Psi_{m n}(r, \phi)=J_{m}\left(\frac{u r}{a}\right) \frac{\cos m \phi}{J_{m}(u)}=\Psi_{i}(r) \cos (m \phi) \\
& \Psi_{m n}(r, \phi)=K_{m}\left(\frac{w r}{a}\right) \frac{\cos m \phi}{K_{m}(w)}=\Psi_{i}(r) \cos (m \phi)
\end{aligned}
$$

for $r<a$ and $r>a$, respectively. The numerical values of $u$ and $w$ are determined from an eigenvalue equation related to the index $n$ [5] and $J_{n}(x)$ and $K_{n}(x)$ are Bessel functions of the first and second kinds [20]. The radial variation of the optical intensities of the lowest order modes is plotted in Fig. 1. From simple considerations of the local gain distribution in such a device, it would be expected that the $\mathrm{LP}_{01}$ mode, that we call the main mode, should preferentially couple to the gain profile arising from a disc geometry contact. We detail explicitly here the case of competition between the $\mathrm{LP}_{01}$ and $\mathrm{LP}_{11}$ degenerate modes, labeled by the indexes $m$ and $s$, respectively. The continuity equation for the local carrier density reads

$$
\begin{aligned}
\frac{\partial N}{\partial t}= & D \nabla^{2} N-B N^{2}-\sum_{i} a_{i} g_{i}(t) I_{i}(t) \Psi_{i}^{2}(r) \\
& +\frac{j_{0}(r, \phi, t)}{e d}
\end{aligned}
$$

where the $i$ index stands for the several modes, $a_{i}=$ $v_{g} /\left[2 \pi L\left\langle\Psi_{i}^{2}\right\rangle\right]$ and

$$
\left\langle\Psi_{i}^{2}\right\rangle=\int_{0}^{\infty} \Psi_{i}^{2}(r) r d r
$$

The modal gains are given by

$$
\begin{aligned}
g_{i}(t)= & \frac{1}{2 \pi\left\langle\Psi_{i}^{2}\right\rangle} \int_{0}^{2 \pi} \int_{0}^{\infty} \Psi_{i}^{2}(r) \\
& \times A_{0}\left[N(r, \phi, t)-N_{t}\right] r d r d \phi
\end{aligned}
$$

as an overlapping of the optical fields with the carrier profile. To complete the formulation of the present model, it is necessary to prescribe the dynamics of the optical intensities as

$$
\frac{d I_{i}}{d t}=\left[v_{g} \Gamma g_{i}(t)-\frac{1}{\tau_{p}}\right] I_{i}+\frac{\beta \bar{N}(t)}{\tau_{n}}+\sqrt{\frac{\beta \bar{N}(t) I_{i}}{\tau_{n}}} \xi_{i}(t)
$$

Here, the same losses are attributed to all modes through the parameter $\tau_{p}$. Of course, differences in modal losses can be anticipated in some circumstances, although the present calculations are not intended to be exhaustive in regard to exploration of dependencies on all parameters. The last terms in (6) take into account spontaneous emission. $\xi_{i}(t)$ is a Gaussian white noise with zero mean and a time correlation given by $\left\langle\xi_{i}(t) \xi_{j}\left(t^{\prime}\right)\right\rangle=2 \delta_{i j} \delta\left(t-t^{\prime}\right)$. We refer to $\beta$ as the parameter coupling spontaneous emission to the several modes. We note, however, that slightly different values should be attributed to each mode in order to more accurately compare with experimental results. The carrier number $\bar{N}(t)$ is given by

$$
\bar{N}(t)=d \int_{0}^{2 \pi} \int_{0}^{\infty} N(r, \phi, t) r d r d \phi .
$$

Device parameters suitable for the VCSEL structure are given in Table I. The above model was used in [8], [9] to investigate steady-state and transient properties of multimode VCSEL's. The spatial dependence of the carrier density, however, does not allow the calculation of interesting quantities such as steady-state optical intensities of the several modes or threshold currents. Moreover, numerical simulation of the complete model requires much computational time. This fact is an important drawback in the evaluation of statistical quantities such as mean turn-on time, time jitter, and side-mode excitation 
TABLE I

Device and Material Parameters

\begin{tabular}{|c|c|c|}
\hline Symbol & Numerical value & Meaning \\
\hline$\epsilon_{1}$ & 12.25 & Dielectric constant of the core region \\
\hline$\epsilon_{2}$ & 11.56 & Dielectric constant of the cladding region \\
\hline$a$ & $3 \mu \mathrm{m}$ & Radius of the core \\
\hline $\mathrm{L}$ & $2 \mu \mathrm{m}$ & Cavity length \\
\hline$d$ & $0.2 \mu \mathrm{m}$ & Active layer thickness \\
\hline$\Gamma$ & 0.1 & Longitudinal confinement factor \\
\hline$e$ & $1.610^{-19} \mathrm{C}$ & Elementary charge \\
\hline $\mathrm{D}$ & $5 \mathrm{~cm}^{2} \mathrm{~s}^{-1}$ & Ambipolar diffusion coefficient \\
\hline B & $10^{-10} \mathrm{~cm}^{3} \mathrm{~s}^{-1}$ & Bimolecular recombination coefficient \\
\hline$N_{t}$ & $1.3310^{18} \mathrm{~cm}^{-3}$ & Transparency carrier density \\
\hline$A_{0}$ & $310^{-16} \mathrm{~cm}^{2}$ & Gain coefficient \\
\hline$\tau_{p}$ & $3 \mathrm{ps}$ & Photon lifetimes \\
\hline$\tau_{n}$ & $2 \mathrm{~ns}$ & Carrier lifetime \\
\hline$\beta$ & $210^{-5}$ & Spontaneous emission factor \\
\hline$v_{g}$ & $0.8610^{8} \mathrm{~ms}^{-1}$ & Group velocity \\
\hline
\end{tabular}

probabilities, because a huge number of turn-on events are needed to perform averages.

In our case, however, the current is injected through a disc, and the modes considered are azimuthally independent. Accordingly, an expansion of the carrier number, dependent only on the radial coordinate and time, can be carried out as in [21] so that

$$
N(r, \phi, t)=\sum_{j=0}^{\infty} N_{j}(t) J_{0}\left(\frac{\gamma_{j} r}{a}\right)
$$

where the numbers $\gamma_{j}$ satisfy $J_{1}\left(\gamma_{j}\right)=0$. Simplification of the equation for the carrier density (3) implies the replacement of the bimolecular recombination term $B N^{2}$ by a linear term $N / \tau_{n}$ including the mean recombination time $\tau_{n}$. The Bessel functions $J_{0}\left(\gamma_{j} r / a\right)$ form a complete set for all functions with vanishing slope in the origin and in $r=a$. The carrier density slope must vanish at the origin when there is azimuthal symmetry and, when the injection current is supplied in a region near the center of the device, a vanishing slope of the carrier density can be assumed at the boundary of the active region [22]. The resulting time evolution of the carrier density components is given by

$$
\frac{d N_{j}}{d t}=C_{j}-\frac{N_{j}}{\tau_{j}}-\sum_{i} \frac{v_{g} g_{i j} g_{i}(t)}{L \pi a^{2} J_{0}^{2}\left(\gamma_{j}\right)} I_{i}
$$

and the time evolution of the optical intensities by

$$
\begin{aligned}
\frac{d I_{i}}{d t}= & {\left[v_{g} \Gamma g_{i}(t)-\frac{1}{\tau_{p}}\right] I_{i}+4 \beta^{\prime} N_{0}(t) } \\
& +\sqrt{4 \beta^{\prime} N_{0}(t) I_{i}} \xi_{i}(t) .
\end{aligned}
$$

In the above equations, new parameters appear

$$
C_{j}(t)=\frac{2}{e d a^{2} J_{0}^{2}\left(\gamma_{j}\right)} \int_{0}^{\infty} j_{0}(r, t) J_{0}\left(\frac{\gamma_{j} r}{a}\right) r d r
$$

which accounts for the driving current of the $j$ component, resulting from the overlapping with the current density profile. $\beta^{\prime}=\beta d \pi a^{2} / 4 \tau_{n}$ stands for the modified noise coefficient and $\tau_{j}$ is given by

$$
\frac{1}{\tau_{j}}=\frac{1}{\tau_{n}}+D\left(\frac{\gamma_{j}}{a}\right)^{2} .
$$

The validity of the expression above relies on the approximation implied by the use of a constant carrier lifetime in place of a bimolecular recombination rate. The modal gain of each mode $g_{i}(t)$ is also simplified in this formalism to

$$
g_{i}(t)=A_{0}\left[\sum_{j=0}^{\infty} g_{i j} N_{j}(t)-N_{t}\right]
$$

where the constants $g_{i j}$ account for the overlapping of the Bessel functions with the optical modes $i$ as

$$
g_{i j}=\frac{1}{\left\langle\Psi_{i}^{2}\right\rangle} \int_{0}^{\infty} \Psi_{i}^{2}(r) J_{0}\left(\frac{\gamma_{j} r}{a}\right) r d r .
$$

In principle, the set of Bessel functions could be replaced by any complete set of functions in the interval $(0, a)$. However, we point to the fact that an arbitrary choice of the set does not lead to independent dynamics of the carrier components $N_{j}(t)$ as in (9). This is mainly due to the circular symmetry of the VCSEL. The present approach is applicable to all azimuthally independent injection current profiles. In spite of its simplicity, the presented model is able to describe phenomena due to carrier diffusion and SHB. As a consequence, it is expected to be useful for studying cases of mode competition during both steady-state and transient evolution. The explicit dependence on the spatial coordinates has completely disappeared. In this way, threshold currents, steady-state optical powers, carrier profiles, and more involved calculations can be carried out analytically. Numerical simulation of a huge number of turnon events become also computationally available. The results given in the rest of this work deal with the multimode rate equations (9) and (10).

\section{Analytical CAlculations}

\section{A. Steady States}

The steady states are obtained from (9) and (10), making zero the time evolution of the variables. For the sake of simplicity, noise effects are not taken into account. When only two modes are considered, four different steady states are possible, depending on the values of the parameters. The first corresponds to the off state where the intensities of both modes are zero and the carrier number components are $N_{j_{s t}}=C_{j} \tau_{j}$. Two states are available with only one lasing mode. The intensity of the lasing mode and the corresponding carrier 
number components read

$$
\begin{aligned}
I_{i_{s t}} & =\frac{L \pi a^{2}}{v_{g} A_{0}\left(g_{t h}-N_{t}\right)} \frac{Y_{i}-g_{t h}}{X_{i}} \\
N_{j_{s t}} & =\tau_{j} C_{j}-\frac{\tau_{j} g_{i j}}{J_{0}^{2}\left(\gamma_{j}\right)} \frac{Y_{i}-g_{t h}}{X_{i}}
\end{aligned}
$$

where

$$
\begin{aligned}
Y_{i} & =\sum_{j=0}^{\infty} g_{i j} \tau_{j} C_{j} \\
g_{t h} & =\frac{1}{\Gamma v_{g} A_{0} \tau_{p}}+N_{t} \\
X_{i} & =\sum_{j=0}^{\infty} \tau_{j}\left[\frac{g_{i j}}{J_{0}\left(\gamma_{j}\right)}\right]^{2} .
\end{aligned}
$$

The existence of these two steady states is conditioned to $I_{i_{s t}}>0$. The last solution gives a bimodal emission with intensities and carrier number components

$$
\begin{aligned}
I_{i_{1}}= & \frac{L \pi a^{2}}{v_{g} A_{0}\left(g_{t h}-N_{t}\right)} \frac{\left(Y_{i_{1}}-g_{t h}\right) X_{i_{2}}-\left(Y_{i_{2}}-g_{t h}\right) W}{\Delta} \\
N_{j_{s t}=} & \tau_{j} C_{j}-\frac{\tau_{j}}{J_{0}^{2}\left(\gamma_{j}\right) \Delta}\left[\left(Y_{i_{1}}-g_{t h}\right)\left(g_{i_{1} j} X_{i_{2}}-g_{i_{2} j} W\right)\right. \\
& \left.+\left(Y_{i_{2}}-g_{t h}\right)\left(g_{i_{2} j} X_{i_{1}}-g_{i_{1} j} W\right)\right]
\end{aligned}
$$

valid for $i_{1}=m, i_{2}=s$ and $i_{1}=s, i_{2}=m$ and where

$$
\begin{aligned}
W & =\sum_{j=0}^{\infty} \tau_{j} \frac{g_{m j} g_{s j}}{J_{0}^{2}\left(\gamma_{j}\right)} \\
\Delta & =X_{m} X_{s}-W^{2} .
\end{aligned}
$$

A linear stability analysis reveals the presence of solutions with two lasing modes. The coexistence of two modes for a definite range of parameters is only possible when hole burning effects are considered, because it takes into account some flexibility with respect to the carrier number profile. Otherwise, the carrier number presents a fixed profile that clearly favors a particular mode and does not allow sharing the gain between different modes. The hole burning effect contained in the continuity equation (3) is still present in the simplified model, through the carrier number expansion given in (8). Although it has little influence during the turn-on process due to small values of the intensity, it is crucial to understand steady-state bimodal operation.

Different treatments have been given in the literature for the study of multimode semiconductor lasers. The transient behavior of nearly single-mode DFB lasers has been widely modeled without considering SHB [12]-[14]. Multimode Fabry-Pérot laser diodes have also been analyzed [11], [23], [24]. The transverse mode structure characteristic of VCSEL's exhibits significant differences with respect to those of conventional edge-emitting semiconductor lasers.

The SMSR can be calculated as

$$
\mathrm{SMSR}=10 \log _{10}\left[\frac{\left\langle I_{m}\right\rangle}{\left\langle I_{s}\right\rangle}\right] .
$$

When both modes are lasing, $\left\langle I_{m}\right\rangle$ and $\left\langle I_{s}\right\rangle$ can be safely substituted by the values of $I_{m_{s t}}$ and $I_{s_{s t}}$, given in (19). In this case, the SMSR value is of a few $\mathrm{dBs}$, because the steadystate emission is actually bimodal. Another situation occurs when the side mode is turned off. $\left\langle I_{m}\right\rangle$ is replaced by $I_{m_{s t}}$ in (15) and the side-mode mean intensity arises only due to noise fluctuations, because the net gain is negative. This mean value is given by

$$
\left\langle I_{s}\right\rangle=\frac{4 \beta^{\prime} N_{0}}{-\gamma v_{g} A_{0}\left[\sum_{j=0}^{\infty} g_{s j} N_{j_{s t}}-g_{t h}\right]}
$$

which generally renders numerical values of SMSR around 30 dBs.

\section{B. Bit Error Rate (BER)}

We are now interested in the statistics of the power partition between the two modes considered during the switching of the laser radiation. At $t=0$, the injection current is suddenly changed from a bias current below threshold to a final value above it, and the current components are changed from $C_{j}^{b}$ to $C_{j}^{\text {on }}$ accordingly. Spontaneous emission noise drives the system from an initial state with a very low photon population to a final state in which the laser emits coherent radiation. Because of spontaneous emission, a random delay exists between the instant at which the threshold gain $g_{t_{t 1} 1}$ is reached and the build-up of the laser radiation. Though the laser usually begins to oscillate in the main mode, noise can occasionally trigger the laser to oscillate on a side mode as well. A qualitative physical explanation of the process is given in order to understand this phenomenon. In rare turn-on events, spontaneous emission, which is a random process, has a very low value at the time the main mode reaches threshold. In this case, the side mode also can have a positive net gain while the laser is in the off state. As a consequence, the side mode also can start lasing depending on the spontaneous emission coupled to each mode.

The statistics of the power partition between the two modes was analyzed in [14] for a DFB laser in terms of the probability density function (PDF) $\Phi\left(I_{m}\right)$ of the main mode intensity $I_{m}$. An analytical expression for $\Phi\left(I_{m}\right)$ was obtained, with the use of a linear approximation neglecting the last term in (9). We have used a similar approach to obtain $P\left(I_{s}>\right.$ $\left.I_{m}\right)$, the probability that the side mode has an intensity larger than that of the main mode when the total intensity is $I_{T}$. This magnitude is analogous to an error rate in optical communication systems. In fact, $P\left(I_{s}>I_{m}\right)$ gives an upper bound for the error rate because the main mode can recover during the evolution to the steady state, and the average power of the main mode over a large enough time interval can be larger than that of the side mode. The value for the BER is thus estimated as

$$
\mathrm{BER}=P\left(I_{s}>I_{m}\right)=\int_{0}^{I_{T} / 2} \Phi\left(I_{m}\right) d I_{m} .
$$

The linear approximation is not accurate when the side mode is highly suppressed and/or the injection current is close to threshold. Calculations can be improved by taking into account 
gain saturation for the side mode due to the main mode [12]. The PDF $\Phi\left(I_{m}\right)$ can be obtained by using the probability current associated with (9) and (10)

$$
\begin{aligned}
\Phi\left(I_{m}\right)= & \int_{0}^{\infty} \frac{1}{\left\langle I_{m}(t)\right\rangle\left\langle I_{s}(t)\right\rangle} \\
& \times\left[\frac{\left\langle\dot{I}_{m}(t)\right\rangle I_{m}(t)}{\left\langle I_{m}(t)\right\rangle}+\frac{\left\langle\dot{I}_{s}(t)\right\rangle\left(I_{T}-I_{m}\right)}{\left\langle I_{s}(t)\right\rangle}\right] \\
& \times \exp \left[-\frac{I_{m}}{\left\langle I_{m}(t)\right\rangle}-\frac{I_{T}-I_{m}}{\left\langle I_{s}(t)\right\rangle}\right] d t
\end{aligned}
$$

where the dots stand for time derivatives and $\left\langle I_{i}(t)\right\rangle$ is the mean intensity value obtained by taking averages in (10) and using the time evolution of the carrier number components solved from (9)

$$
N_{j}(t)=\tau_{j} C_{j}^{\mathrm{on}}+\tau_{j}\left(C_{j}^{b}-C_{j}^{\mathrm{on}}\right) e^{-t / \tau_{j}} .
$$

The steepest descent method has been employed to develop the integral arising from the calculations $\int_{t}^{t_{i_{11}}}\left(\sum_{j=0}^{\infty} g_{i j} N_{j}(t)-\right.$ $\left.g_{\mathrm{th}}\right) d t$ around the threshold time $t=t_{i_{\mathrm{tl}}}$, in which $\sum_{j=0}^{\infty} g_{i j} N_{j}\left(t=t_{i_{\mathrm{th}}}\right)=g_{\mathrm{th}}$. As a result, $\left\langle I_{i}(t)\right\rangle$ can be written as

$$
\begin{aligned}
\left\langle I_{i}(t)\right\rangle= & \left\langle I_{i_{\mathrm{th}}}(t)\right\rangle \exp \left(\Gamma v_{g} A_{0} \sum_{j=0}^{\infty} g_{i j} \tau_{j}\left(C_{j}^{o n}-C_{j}^{t_{\mathrm{t}_{\mathrm{ll}}}}\right)\right. \\
& \left.\times\left\{\left(t-t_{i_{\mathrm{th}}}\right)-\tau_{j}\left[1-e^{\left(t-t_{i_{\mathrm{th}}}\right) / \tau_{j}}\right]\right\}\right)
\end{aligned}
$$

where $C_{j}^{t_{i_{\mathrm{th}}}}=N_{j}\left(t=t_{i_{\mathrm{th}}}\right) / \tau_{j}$ and

$$
\begin{aligned}
&\left\langle I_{i_{\mathrm{thl}}}(t)\right\rangle= \frac{2 \beta^{\prime} \sqrt{\pi} \tau_{0} C_{0}^{t_{i \mathrm{th}}}}{\sqrt{v_{i}}}\left\{\Phi\left[\sqrt{v_{i}}\left(t-t_{i_{\mathrm{tll}}}\right)\right]+\Phi\left[\sqrt{v_{i}} t_{i_{\mathrm{tll}}}\right]\right\} \\
&+ \frac{4 \beta^{\prime} \tau_{0} C_{0}^{b}}{\Gamma v_{g} A_{0} \sum_{j=0}^{\infty} g_{i j} \tau_{j}\left(C_{j}^{t_{i \mathrm{t}_{1}}}-C_{j}^{b}\right)} \\
& \times \exp \left\{\Gamma v_{g} A_{0} \sum_{j=0}^{\infty} g_{i j} \tau_{j}\left(C_{j}^{\mathrm{on}}-C_{j}^{t_{i \mathrm{th}}}\right)\right. \\
&\left.\times\left[t_{i_{\mathrm{th}}}+\tau_{j}\left(1-e^{t_{i_{\mathrm{th}}} / \tau_{j}}\right)\right]\right\}
\end{aligned}
$$

with $C_{j}^{b}=N_{j}(t=0) / \tau_{j}, v_{i}=\Gamma v_{g} A_{0} / 2 \sum_{j=0}^{\infty} g_{i j}\left(C_{j}^{\text {on }}-\right.$ $\left.C_{j}^{t_{i} \mathrm{th}_{1}}\right)$ and $\Phi(x)$ is the error function [20]. From (24) and (25), an analytical expression for the BER is obtained.

\section{RESUlTS AND DisCUSSION}

In this section, the steady-state and transient behavior of the VCSEL is discussed, considering the geometry and material parameters characteristic of these devices. The analysis is based on the analytical results obtained in Section III. The

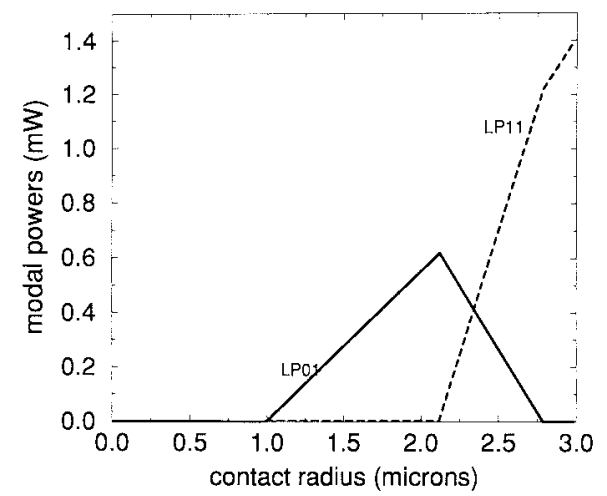

Fig. 2. Steady-state modal powers $\mathrm{LP}_{01}$ (solid line) and $\mathrm{LP}_{11}$ (dashed line) as a function of the contact radius (15) and (19). The current density is $20 \times 10^{7} \mathrm{~A} / \mathrm{m}^{2}$.

several analytical approximations leading to the expression for the BER (24) are tested by means of numerical simulations of the stochastic rate equations (9) and (10). A convenient number of turn-on events have been considered in the averaging process in order to guarantee statistical errors lower than $10 \%$. In the expansion for the carrier density (8), only terms up to $j=3$ have been kept in all the calculations and numerical simulations performed. This simplification is justified by considering the decreasing overlapping of the optical modes with the Bessel functions of increasing order. The resulting low numerical values of the constants $g_{i j}$ give an almost vanishing value of the contribution of the $N_{j}$ component.

\section{A. Contact Geometry}

The disc geometry of the contact clearly favors the appearance of the main $\mathrm{LP}_{01}$ mode. This is shown in Fig. 2, where the modal powers (15) and (19) are plotted as a function of the contact radius. The current density has been kept to a constant value of $20 \times 10^{7} \mathrm{~A} / \mathrm{m}^{2}$. Laser emission is monomode up to $2.1 \mu \mathrm{m}$. Beyond this value of the contact radius, the secondary mode $\mathrm{LP}_{11}$ mode appears as a consequence of SHB.

Turn-on events have been considered by suddenly switching on the current density from a below threshold value of 4 $\times 10^{7} \mathrm{~A} / \mathrm{m}^{2}$ to the final value $20 \times 10^{7} \mathrm{~A} / \mathrm{m}^{2}$. The main mode is turned-on with a probability given by $\Phi\left(I_{M}\right)$ (25), which is plotted in Fig. 3 as a solid line when the contact radius is set to $2.3 \mu \mathrm{m} .10^{4}$ turn-on events were considered to perform the histogram, with a remarkable agreement with the analytical results. This agreement justifies the evaluation of the BER from the analytical expression (24), plotted in Fig. 4 as a function of the contact radius. The reference value for the intensity has been set to $I_{T}=I_{m}+I_{s}=5487$, corresponding to an output power of $0.1 \mathrm{~mW}$. Stars in that figure indicate the results of averaged numerical simulations. We would like to stress the importance of having an analytical expression for the BER because, otherwise, predictions on the performance of the device during gain-switched operation would be limited to BER's larger than $10^{-4}$ due to the tremendous computational time required.

Fig. 4 reveals that the BER increases with increasing values of the contact radius, due to the fact that monomode emission 


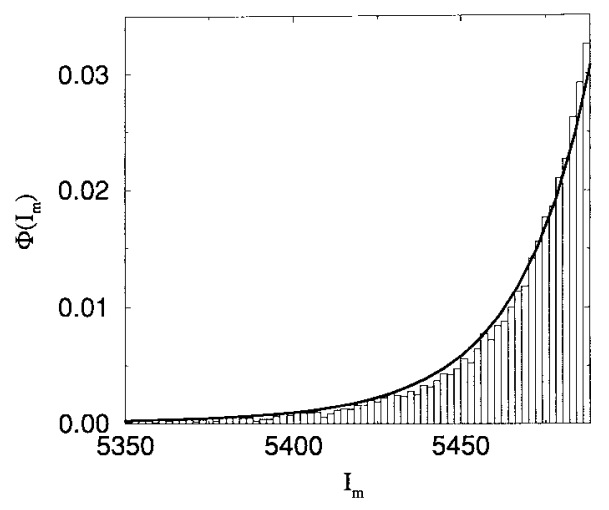

Fig. 3. PDF of the main mode $\mathrm{LP}_{01}$ intensity at the laser turn-on $\left(I_{T}=I_{m}+I_{s}=5487\right.$, corresponding to an output power of $0.1 \mathrm{~mW}$ ). The histogram performed after $10^{4}$ numerically simulated turn-on events is compared with the analytical calculations (25), plotted as a solid line. Here the contact radius is $2.3 \mu \mathrm{m}$ and the on current density is $20 \times 10^{7} \mathrm{~A} / \mathrm{m}^{2}$.

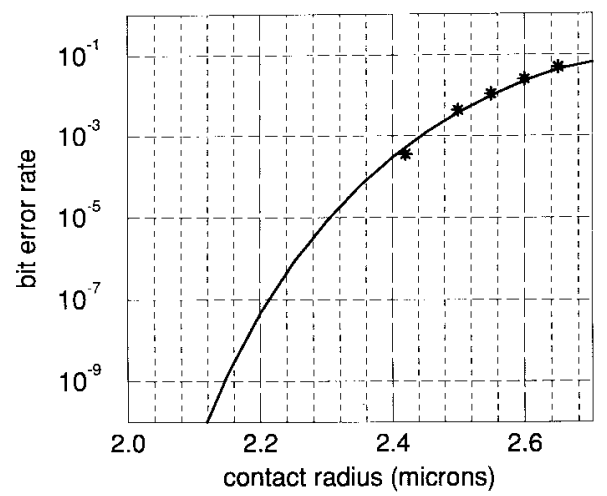

Fig. 4. BER as a function of the contact radius. The stars indicate numerically simulated BER's, with a $10 \%$ relative error. The solid line includes the calculations given in (24).

is lost. There is a clear tradeoff between the maximum power attainable and monomode emission in the main mode.

\section{B. $S H B$}

The transverse mode structure in the lasing regime is essentially defined by the relevant modal gains which, in turn, are determined both by the mode field distributions and the spatial distribution of the carriers in the device active region. It has been numerically shown [8], [9] that the interplay between SHB and the modal field distribution may result in two principal steady-state behaviors. In the first case where the modal fields are spatially separated, it is found that the modes may effectively share the available gain resulting in multimode operation. On the other hand, when the modes overlap significantly and thus occupy essentially the same part of the device active region, then there is strong competition between the modes which may result in just one mode dominating. Which of the two modes dominates is determined simply by which has the highest modal gain. In our case, the modes $\mathrm{LP}_{01}$ and $\mathrm{LP}_{11}$ (see Fig. 1) present a spatial overlapping that is not enough to prevent multimode emission during steady-state operation. This is illustrated in Fig. 5 as a function of the current density supplied to the VCSEL (the contact radius here is $2.3 \mu \mathrm{m}$ ). The threshold current is at $6 \times$

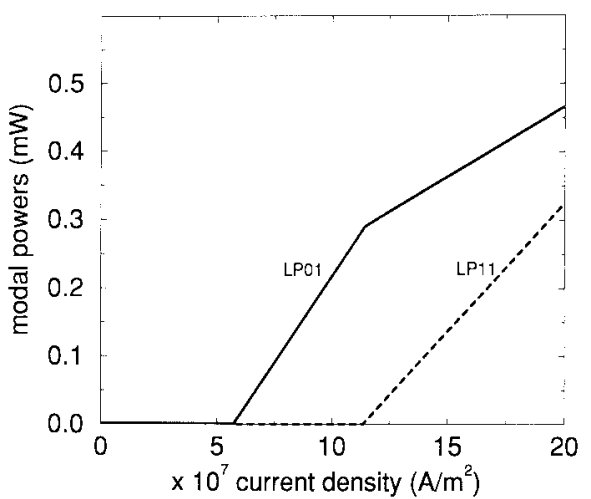

Fig. 5. Steady-state modal powers $\mathrm{LP}_{01}$ (solid line) and $\mathrm{LP}_{11}$ (dashed line) as a function of the current density (15), (19). The contact radius here is 2.3 $\mu \mathrm{m}$.

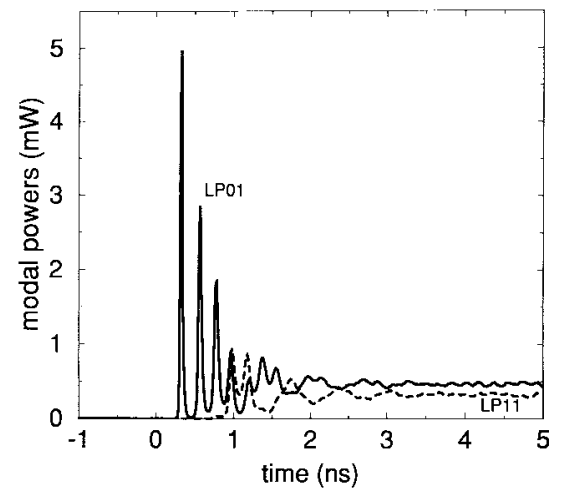

Fig. 6. Typical time trace of the modal powers $\mathrm{LP}_{01}$ (solid line) and $\mathrm{LP}_{11}$ (dashed line) after a current switch at $t=0$. The contact radius here is $2.3 \mu \mathrm{m}$.

$10^{7} \mathrm{~A} / \mathrm{m}^{2}$. Monomode emission in the main mode is achieved up to $12 \times 10^{7} \mathrm{~A} / \mathrm{m}^{2}$ where the secondary mode starts to lase. Sharing of the available gain is such that the total power has a linear dependence with the current density independently of the nature of the modal emission.

Steady-state emission in the secondary mode $\mathrm{LP}_{11}$ is due to SHB present at high output powers. Without considering these SHB effects, lasing would always occur at the main mode, which present the best overlapping with the carrier profile in the off state. A typical time trace of the modal powers following a current switch is shown in Fig. 6. The threshold time $t_{i_{\mathrm{thl}}}$ is in all cases shorter for the main mode. Accordingly, the $\mathrm{LP}_{01}$ mode usually starts lasing emission, in the figure shown with three power overshoots. When a significant number of photons are present in the laser microcavity, a hole is burned in the carrier profile which leads to the emission in the secondary mode $\mathrm{LP}_{11}$. The situation can be clearly visualized in Fig. 7, where the analytical expansions (8) for the carrier number are plotted in the initial and final steady states. With the final carrier profile, both modes are able to lase by sharing the available gain.

In DFB lasers, monomode emission during steady state is a requirement for monomode emission during large signal modulation or gain-switching. Usually a SMSR larger than $30 \mathrm{~dB}$ is needed in order to guarantee single mode during the transient, with a BER lower than $10^{-9}$. We have shown 


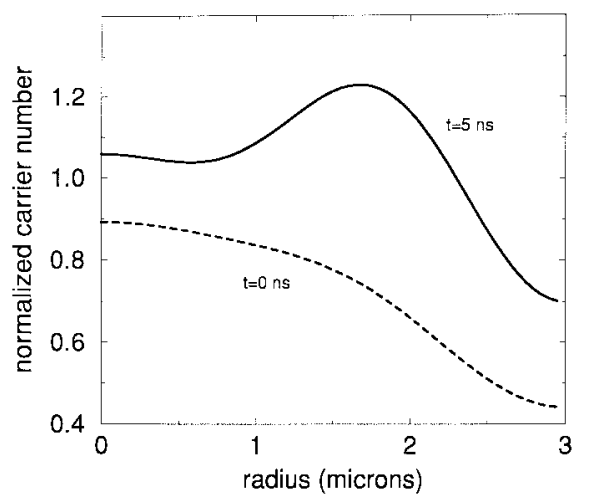

Fig. 7. Normalized carrier number before (dashed line) and after (solid line) the laser switch-on (8), where the SHB is clearly appreciated. The contact radius here is $2.3 \mu \mathrm{m}$.

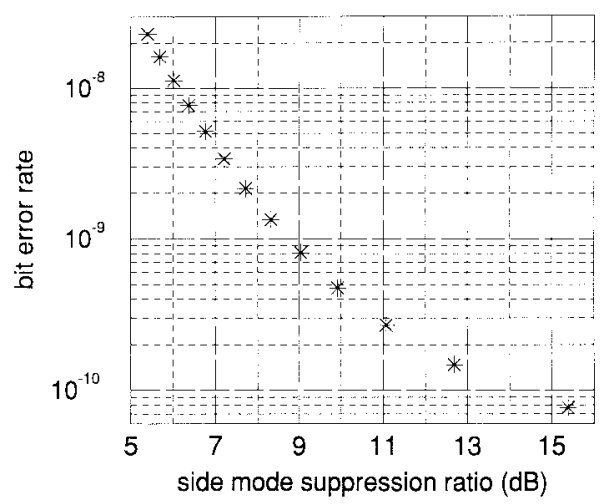

Fig. 8. BER (24) as a function of the SMSR (22) at different current densities, for a contact radius of $2.3 \mu \mathrm{m}$. BER's as low as $10^{-9}$ are possible with poor SMSR's of $9 \mathrm{dBs}$.

that, in VCSEL's, multimode steady-state emission appears after a monomode turn-on due to SHB. As a result, very low BER's can be achieved even with poor SMSR's in the on state. This remarkable result points to an optimal performance of VCSEL's as modulated optical emitters in fiber optic communication systems working at high bit rates. Numerical examples are given in Fig. 8, where the contact radius has been set to $2.3 \mu \mathrm{m}$ and the current density value has been scanned. BER's lower than $10^{-9}$ are easily achieved even with SMSR's as poor as $10 \mathrm{~dB}$.

\section{Carrier Diffusion}

Another physical effect that is expected to modify the performance of the VCSEL is carrier diffusion. Carrier diffusion is responsible for the carrier profile both during the off and on steady states. It was shown in [9] that this effect helps to maintain modal discrimination and increase the threshold currents. This observation is illustrated in Fig. 9, where the density current is $20 \times 10^{7} \mathrm{~A} / \mathrm{m}^{2}$ and the contact radius is $2.3 \mu \mathrm{m}$. The diffusion parameter of $5 \mathrm{~cm}^{2} / \mathrm{s}$ characteristic of the particular VCSEL structure permits multimode lasing, but larger values of this parameter, beyond $9 \mathrm{~cm}^{2} / \mathrm{s}$, only allow monomode emission in the main mode. Carrier diffusion drastically modifies the transient multimode operation of the laser, as can be seen in Fig. 10. Slight changes in the numerical

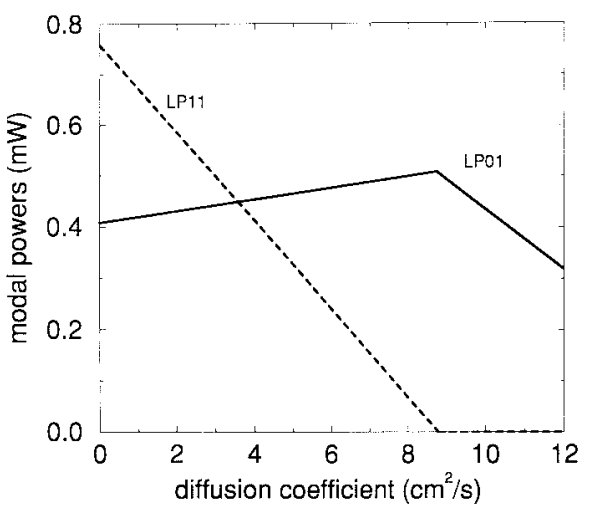

Fig. 9. Steady-state modal powers $\mathrm{LP}_{01}$ (solid line) and $\mathrm{LP}_{11}$ (dashed line) as a function of the diffusion coefficient (15) and (19). The current density is $20 \times 10^{7} \mathrm{~A} / \mathrm{m}^{2}$ and the contact radius $2.3 \mu \mathrm{m}$.

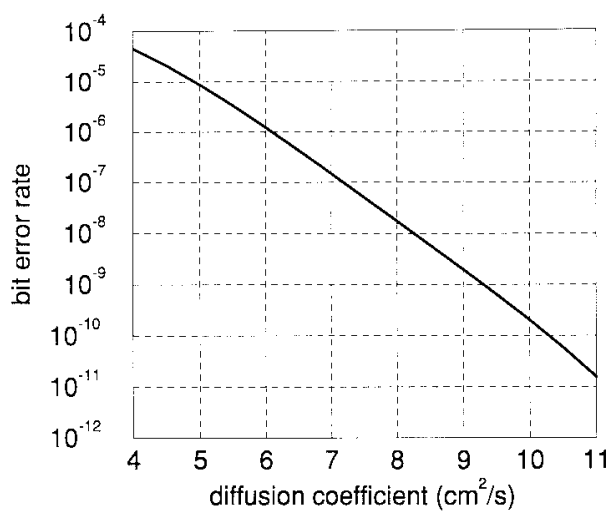

Fig. 10. BER (24) as a function of the diffusion coefficient $D$. In this plot, a strong sensitivity with respect to diffusive effects can be appreciated during the laser turn-on.

value of the diffusion parameter lead to important changes, of several orders of magnitude, in the BER.

The multimode rate equations (9) and (10) derived in Section III allow for a discussion of the role played by carrier diffusion. The diffusion parameter contributes to modify the numerical value of $\tau_{n}$ (12), to give different recombination times for the carrier components $N_{j}(t)$. An expansion of (26) reveals that the time evolution of the carrier components, and consequently the threshold times, are not modified in a first-order approximation by changes in $\tau_{j}$. Accordingly, we attribute the large sensitivity of BER to carrier diffusion to different initial carrier profiles and thus modal gains.

\section{Spontaneous Emission}

During steady-state operation, spontaneous emission noise is known to increase power and frequency fluctuations, and also the relative intensity noise figure. It is also responsible for triggering the turn-on process of solitary lasers. Long time tails appear in the PDF of turn-on times due to the randomness of the seeding process. There is a delay between the time taken by the two modes to reach the threshold gain. Usually, the noise is able to trigger the oscillation in the main mode (the first to reach the threshold gain) before the secondary mode experiences a positive net gain. In rare turn-on events lying in the long time tails of the PDF, however, laser emission can 
start in the secondary mode. This situation occurs because the late turn-on event has lasted enough to permit net gain for the two modes simultaneously.

Here an interesting result becomes evident. An increase of the spontaneous emission noise enhances monomode operation during the transient following the current switch. In effect, increasing noise leads to early turn-on events, and the appearance of side modes with higher threshold times can be almost completely canceled.

\section{CONCLUSION}

A simplified analytical approach has been presented and applied to the turn-on process of a multimode VCSEL. The model incorporates the influence of carrier diffusion and SHB. Analytical expressions for the steady-state modal powers, SMSR, and bit error rate have been derived. Numerical simulations are shown in agreement with the obtained results. The time traces for the modal powers are also in qualitative agreement with experimental results reported recently. A detailed analysis points to a better single-mode performance of VCSEL's compared to that of multimode edge-emitting lasers. The influence of contact geometry, SHB, carrier diffusion, and spontaneous emission is discussed.

\section{ACKNOWLEDGMENT}

The authors acknowledge helpful discussions with A. Valle, L. Pesquera, and J. Sarma.

\section{REFERENCES}

[1] K. Iga, "Surface emitting lasers," Opt. Quantum Electron., vol. 24, pp. S97-S104, 1992.

[2] M. A. Fisher, Y. Z. Huang, A. J. Dann, D. J. Elton, M. J. Harlow, S. D. Perrin, J. Reed, I. Reid, H. J. Wickes, and M. J. Adams, "1.5 $\mu \mathrm{m}$ verticalcavity surface-emitting lasers," in Tech. Dig. OSA Semiconductor Lasers Top. Meet., 1995, Paper TuD2.

[3] F. Prati, A. Tesei, and L. A. Lugiato, "Stable states in surfaceemitting semiconductor lasers," Chaos, Solitons and Fractals, vol. 4, pp. 1637-1654, 1994

[4] H. Li, T. L. Lucas, J. G. McInerney, and R. A. Morgan, "Transverse modes and patterns in electrically pumped vertical cavity surface emitting semiconductor lasers," in Tech. Dig. CLEO-Europe Conf., 1994 Paper CWD5.

[5] C. H. Chong and J. Sarma, "Lasing mode selection in vertical cavity surface emitting laser diodes," IEEE Photon. Technol. Lett., vol. 5, pp. 761-763, 1993

[6] _ "Self-consistent calculations of two-dimensional carrier distribution and modal gain of lasing modes in cylindrical VCSEL's," in OE/LASE 94, Physics and Simulation of Optoelectronic Devices II, SPIE Tech. Conf. 2146, 1994, Paper 43.

[7] G. R. Hadley, K. L. Lear, M. E. Warren, K. D. Choquette, J. W. Scott, and S. W. Corzine, "Comprehensive numerical modeling of vertical cavity surface-emitting lasers," IEEE J. Quantum Electron., vol. 32, pp. 607-616, 1996.

[8] A. Valle, J. Sarma, and K. A. Shore, "Dynamics of transverse mode competition in vertical cavity surface emitting semiconductor lasers," Opt. Commun., vol. 115, pp. 297-302, 1995.

[9] , "Spatial hole-burning effects on the dynamics of vertical cavity surface emitting semiconductor lasers," IEEE J. Quantum Electron., vol. 31, pp. 1423-1431, 1995.
[10] O. Buccafusca, J. L. A. Chilla, J. J. Rocca, S. Feld, C. Wilmsen, V. Morozov, and R. Leibenguth, "Transverse mode dynamics in vertical cavity surface emitting lasers excited by fast electrical pulses," Appl. Phys. Lett., vol. 68, pp. 590-592, 1996.

[11] A. D’Ottavi, A. Mecozzi, P. Spano, and P. Piazolla, "Time jitter in multimode Fabry-Perot laser diodes," Appl. Phys. Lett., vol. 53, pp. 2362-2364, 1988.

[12] A. Valle, P. Colet, L. Pesquera, and M. San Miguel, "Transient multimode statistics in almost single-mode semiconductor-lasers," Proc. Inst. Elect. Eng., part J, vol. 140, pp. 237-242, 1993.

[13] A. Valle, C. R. Mirasso, and L. Pesquera, "Mode partition noise of nearly single-mode semiconductor-lasers modulated at GHz rates," IEEE J. Quantum Electron., vol. 31, pp. 876-885, 1995.

[14] A. Mecozzi, A. Sapia, P. Spano, and G. P. Agrawal, "Transient multimode dynamics in nearly single-mode lasers," IEEE J. Quantum Electron., vol. 27, pp. 332-343, 1991 and Jpn. J. Appl Phys, vol. 30, pp. 3879-3882, 1991.

[15] A. Valle, J. Sarma, and K. A. Shore, "Secondary pulsations driven by spatial hole burning in vertical cavity surface emitting laser diodes," $J$. Opt. Soc. Amer. B, vol. 12, pp. 1741-1746, 1995.

[16] J. Dellunde, A. Valle, and K. A. Shore, "Transverse mode selection in external cavity vertical cavity surface emitting laser diodes," J. Opt. Soc. Amer. B, vol. 13, pp. 2477-2483, 1996.

[17] M. Ogura, S. Fujii, T. Okada, M. Mori, T. Asaka, and H. Iwanu, "Transverse mode characteristics of DBR-surface emitting laser with buried heterostructure," Jpn. J. Appl. Phys., vol. 30, pp. 3879-3882, 1991.

[18] F. Koyama, K. Morito, and K. Iga, "Intensity noise and polarization stability of GaAlAs-GaAs surface emitting lasers," IEEE J. Quantum Electron., vol. 27, pp. 1410-1416, 1991.

[19] M. S. Sodha and A. K. Ghatak, Inhomogeneous Optical Waveguides. New York: Plenum, 1977, pp. 61-71.

[20] M. Abramowitz and I. A. Stegun, Eds., Handbook of Mathematical Functions. New York: Dover, 1972

[21] K. Moriki, H. Nakahara, T. Hattori, and K. Iga, "Single transverse mode condition of surface-emitting injection lasers," Electron. Commun. Japan, Part 2, vol. 71, pp. 81-90, 1988.

[22] J. Dellunde, M. C. Torrent, J. M. Sancho, and K. A. Shore, "Transverse multimode dynamics of gain-switched VCSEL's," in OSA Integrated Photonics Research Conf., 1996, Paper ITuE2.

[23] K. Otsuka, M. Georgiou, and P. Mandel, "Intensity fluctuations in multimode lasers with spatial hole burning," Jpn. J. Appl. Phys., vol. 31, pp. 1250-1252, 1992.

[24] P. Mandel, M. Georgiou, K. Otsuka, and D. Pieroux, "Transient and modulation dynamics of a multimode Fabry-Pérot," Opt. Commun., vol. 100, pp. 341-350, 1993.

J. Dellunde, photograph and biography not available at the time of publication

M. C. Torrent, photograph and biography not available at the time of publication.

J. M. Sancho, photograph and biography not available at the time of publication

K. A. Shore, photograph and biography not available at the time of publication. 